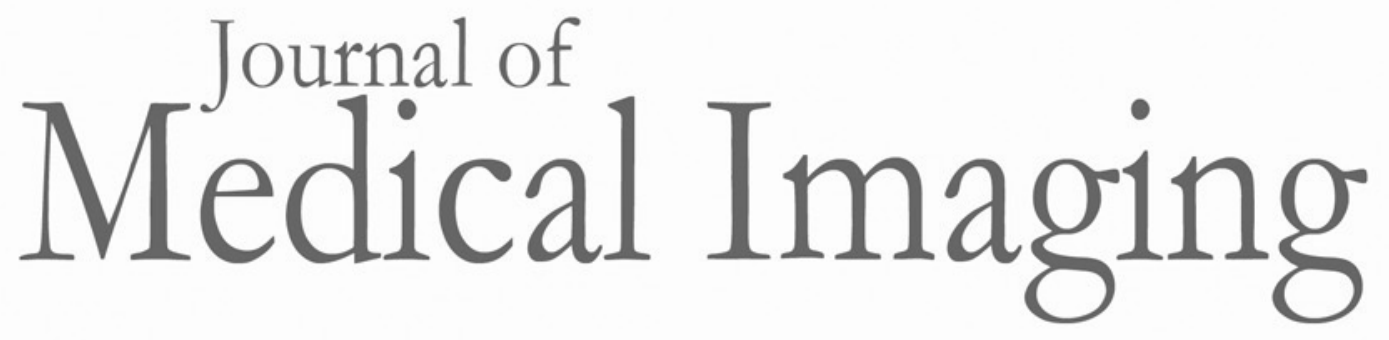

\title{
Erratum: Measuring temporal stability of positron emission tomography standardized uptake value bias using long-lived sources in a multicenter network
}

Darrin Byrd

Rebecca Christopfel

Grae Arabasz

Ciprian Catana

Joel Karp

Martin A. Lodge

Charles Laymon

Eduardo G. Moros

Mikalai Budzevich

Sadek Nehmeh

Joshua Scheuermann

John Sunderland

Jun Zhang

Paul Kinahan 


\title{
Erratum: Measuring temporal stability of positron emission tomography standardized uptake value bias using long-lived sources in a multicenter network
}

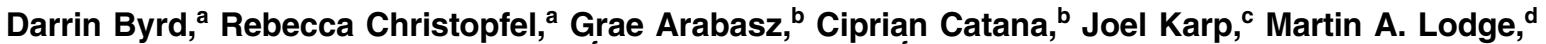 \\ Charles Laymon, ${ }^{\mathrm{e}}$ Eduardo G. Moros, ${ }^{\mathrm{f}}$ Mikalai Budzevich, ${ }^{\mathrm{f}}$ Sadek Nehmeh, ${ }^{\mathrm{g}}$ Joshua Scheuermann, ${ }^{\mathrm{c}}$ \\ John Sunderland, ${ }^{\mathrm{h}}$ Jun Zhang, ${ }^{i}$ and Paul Kinahan ${ }^{\mathrm{a}}$ \\ aUniversity of Washington, Department of Radiology, Seattle, Washington, United States \\ ${ }^{b}$ Athinoula A. Martinos Center for Biomedical Imaging, Department of Radiology, Massachusetts General Hospital and Harvard Medical School, \\ Charlestown, Massachusetts, United States \\ 'University of Pennsylvania, Department of Radiology, Philadelphia, Pennsylvania, United States \\ dJohns Hopkins University, Department of Radiology and Radiological Science, Baltimore, Maryland, United States \\ eUniversity of Pittsburgh, Presbyterian University Hospital, Department of Radiology, Pittsburgh, Pennsylvania, United States \\ f'Moffitt Cancer Center, Tampa, Florida, United States \\ 'Weill Cornell Medical College, Department of Radiology, New York, United States \\ hUniversity of lowa, Department of Radiology, lowa City, lowa, United States \\ 'The Ohio State University, Department of Radiology, Columbus, Ohio, United States
}

[DOI: 10.1117/1.JMI.6.1.019801]

This article [J. Med. Imag. 5(1), 011016 (2018), doi: 10.1117/1 .JMI.5.1.011016] was originally published online on 4 January 2018 with the following error. The Pearson correlation coefficients were not computed and reported in a consistent fashion, most notably in Table 10, where values labeled " $r$ " were instead the values of $r$ without appropriate squaring. Figure 8 contained a similar inconsistency, though not all values were affected.

Original (erroneous) and corrected correlation coefficients from Table 10 are enlisted here with their corresponding measurements:

\begin{tabular}{|c|c|c|c|}
\hline \multicolumn{2}{|c|}{ Original (erroneous) } & \multicolumn{2}{|c|}{ Corrected } \\
\hline Measurements & Correlation coefficient & Measurements & Correlation coefficient $r^{2}$ \\
\hline 12 & 0.966 & 12 & 0.934 \\
\hline 12 & 0.956 & 12 & 0.915 \\
\hline 10 & 0.934 & 10 & 0.872 \\
\hline 7 & 0.867 & 7 & 0.752 \\
\hline 10 & 0.583 & 10 & 0.340 \\
\hline 20 & 0.993 & 20 & 0.985 \\
\hline 15 & 0.851 & 15 & 0.725 \\
\hline 8 & 0.866 & 8 & 0.749 \\
\hline 15 & 0.378 & 15 & 0.143 \\
\hline 6 & 0.976 & 6 & 0.953 \\
\hline 8 & 0.594 & 8 & 0.353 \\
\hline 3 & 0.387 & 3 & 0.150 \\
\hline 8 & 0.944 & 8 & 0.892 \\
\hline \multirow[t]{2}{*}{$10.3 \pm 4.3$} & $0.792 \pm 0.216$ & 11 & 0.002 \\
\hline & & $10.4 \pm 4.2$ & $0.626 \pm 0.337$ \\
\hline
\end{tabular}

C. 2019 Society of Photo-Optical Instrumentation Engineers (SPIE) 
The original and the corrected Figure 8 are depicted below:

Original

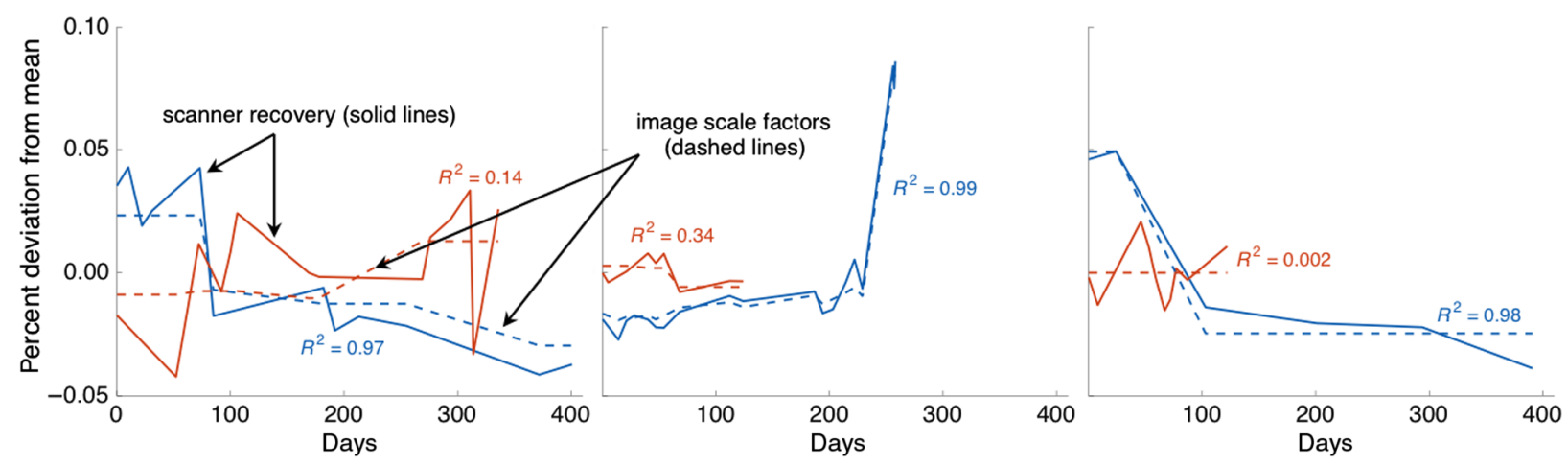

\section{Corrected}
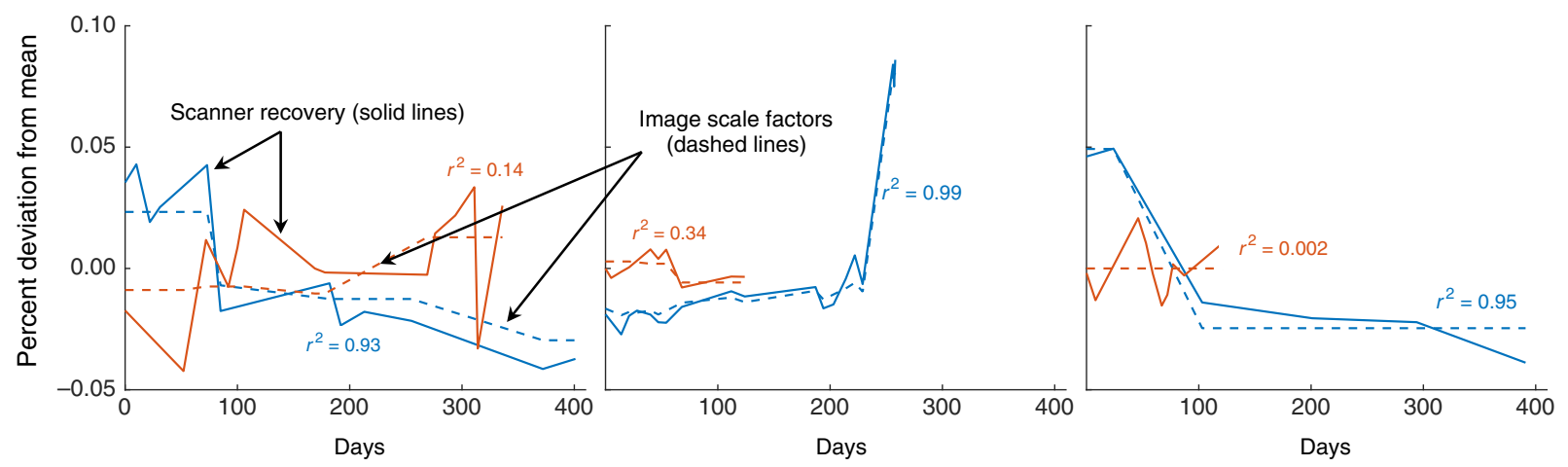

This article was corrected online on 23 March 2019.

It appears correctly in print. 